\title{
Tricritical properties of polymer chains in a poor solvent
}

\author{
B. Duplantier \\ Division de la Physique, Service de Physique Théorique, CEN Saclay, B.P. no 2, 91190 Gif sur Yvette, France
}

(Reçu le 22 avril 1980, accepté le 4 juillet 1980)

\begin{abstract}
Résumé. - Les propriétés des polymères en solution dans le domaine $\theta$ sont déduites d'une théorie lagrangienne, semblable à celle d'un point tricritique en mécanique statistique. Des lois d'échelles nouvelles logarithmiques sont établies pour la pression osmotique et le rayon moyen des chaînes. La courbe de coexistence de chaînes infinies est déterminée. La théorie tricritique prédit pour cette courbe une pente nulle à l'origine.
\end{abstract}

\begin{abstract}
The properties of polymer solutions in the $\theta$-domain are deduced from a Lagrangian theory, similar to that of a tricritical point in statistical mechanics. New logarithmic scaling laws are established for the osmotic pressure and the mean radius of the chains. The coexistence curve for infinite chains is determined. The tricritical theory predicts for this curve a zero slope at the origin.
\end{abstract}

1. Introduction. - The critical properties of long polymer chains in a good solvent $[1,2]$ have been predicted successfully from Lagrangian field theories of the Wilson type. However, chains in a poor solvent $(\theta$-domain) have different renormalization properties, related to tricritical phenomena [3]. A general study of tricriticality in a $\theta$-solvent, after that of criticality in a good solvent, is thus necessary. Some partial results have been obtained in this direction $[4,5,6,7]$, mainly restricted to the single chain case. We establish here the scaling properties of chain solutions in the whole $\theta$-domain. For this purpose, we represent a grand ensemble of chains [2] in a poor solvent by a tricritical Lagrangian field theory with an external field [8]. Its properties are obtained from a renormalization group method $[9,10]$, the details of which will be given elsewhere.

2. Lagrangian theory. - Let us consider continuous polymer chains in a large box of volume $V$. The chains are characterized by a parameter $S$, their Brownian surface, defined by $\left(R^{2}\right)_{\text {Brownian }}=d . S$ ( $d=$ space dimension). For chains made of $N$ links : $S=N l^{2}$, where $l$ is a length.
In a poor solvent, the scaling properties of chains can be determined by considering two effective interactions [3], which we will take to be local : the pair interaction $V(x-y)=g \delta(x-y)$ and the threemonomer interaction

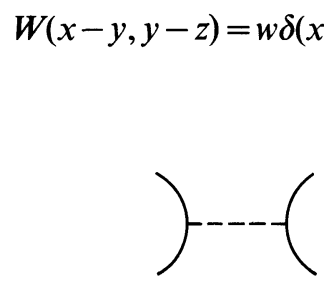

a)
(Fig. 1) .

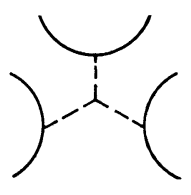

b)
Fig. 1. - The two-monomer $(a)$ and three-monomer $(b)$ interaction vertices.

A cut-off is also necessary : a minimal Brownian surface $s$ is supposed to separate two interaction points along a same continuous chain.

A grand ensemble of polymer chains [2] can then be described by the action [8] :

$$
\begin{aligned}
A\{\varphi\}=\int \mathrm{d}^{d} x\left\{\frac{1}{2} \sum_{i, j}\left[\partial_{i} \varphi^{j}(x)\right]^{2}+\frac{1}{2} a \sum_{j}\left[\varphi^{j}(x)\right]^{2}\right. & +\frac{1}{8} g \sum_{j, l}\left[\varphi^{j}(x)\right]^{2}\left[\varphi^{l}(x)\right]^{2}+ \\
& \left.+\frac{1}{2^{3} 3 !} w \sum_{j, l, m}\left[\varphi^{j}(x)\right]^{2}\left[\varphi^{l}(x)\right]^{2}\left[\varphi^{m}(x)\right]^{2}+h \varphi^{1}(x)\right\}
\end{aligned}
$$


$\varphi^{j}(x)$ is a $n$-component field, $j=1, \ldots, n . a$ is a chemical potential associated with the Brownian surface $S$ of the chains. $h^{2}$ is a fugacity associated with the number of chains. For polymer chains $: n \rightarrow 0$ [1].

The free energy of the grand ensemble is then $[2,8]$

$$
W(h)=V^{-1} \ln \int D^{n}\{\varphi\} \exp (-A\{\varphi\}) .
$$

The grand potential $\Gamma(f)$ is the Legendre transform of $W(h)$, where $f$ and $h$ are conjugate variables. Then the chain concentration $C_{\mathrm{p}}$, the monomer concentration $C=C_{\mathrm{m}} l^{2}$, and the osmotic pressure are given by [2]

$$
\begin{gathered}
C=C_{\mathrm{p}} S=2 \frac{\partial}{\partial a} \Gamma(f) ; \\
C_{\mathrm{p}}=\frac{1}{2} f \frac{\partial}{\partial f} \Gamma(f) ; \\
\Pi \beta=f \frac{\partial}{\partial f} \Gamma(f)-\Gamma(f),
\end{gathered}
$$

where $S$ is the mean surface per chain. The mean square end to end distance of a chain is

$$
R^{2}=2 d \frac{\partial \Gamma_{\mathrm{T}}^{(2)}}{\partial k^{2}}(0 ; f) / \Gamma_{\mathrm{T}}^{(2)}(0 ; f)
$$

where $\Gamma_{\mathbf{T}}^{(2)}(k ; f)$ is the transverse vertex function [11], in the momentum space, associated with (1).

At a tricritical point, the vertex functions of rank 2 and 4 vanish at zero momenta. One can check that it corresponds to an infinite chain system with a vanishin second virial coefficient [3]. The excluded volume parameter has there the value $g_{\theta}\left(g_{\theta}<0\right)$. $b=g-g_{\theta}$ measures the deviations from the $\theta$-line. For a temperature $T$ near $\theta$ :

$$
b=g-g_{\theta} \cong A\left(\frac{T-\theta}{\theta}\right),
$$

where $A$ is a coefficient.

$\Gamma(f)$ can be calculated from (1) by a loop-expansion. This reads, to the one-loop order [12]

$$
\begin{aligned}
\Gamma(f) & =\Gamma_{0}(f)-\frac{1}{12 \pi}\left[\left(\frac{\partial^{2} \Gamma_{0}(f)}{\partial f^{2}}\right)^{3 / 2}-\left(\frac{1}{f} \frac{\partial \Gamma_{0}(f)}{\partial f}\right)^{3 / 2}\right] \\
\Gamma_{0}(f) & =\frac{1}{2} a f^{2}+\frac{1}{8} g f^{4}+\frac{1}{48} w f^{6} .
\end{aligned}
$$

We set $n=0$. To compute (3), one needs also the one-loop relations :

$$
\begin{gathered}
\Gamma_{\mathrm{T}}^{(2)}(0 ; f)=\frac{1}{f} \frac{\partial}{\partial f} \Gamma(f) \\
\frac{\partial \Gamma_{\mathrm{T}}^{(2)}}{\partial k^{2}}(0 ; f)=1+\frac{1}{12 \pi} f^{-2}\left[\left(\frac{\partial^{2} \Gamma_{0}(f)}{\partial f^{2}}\right)^{1 / 2}-\left(\frac{1}{f} \frac{\partial \Gamma_{0}(f)}{\partial f}\right)^{1 / 2}\right]\left[\left(\frac{1}{f} \frac{\partial}{\partial f} f \frac{\partial \Gamma_{0}(f)}{\partial f}\right)\right]^{-1} .
\end{gathered}
$$

The loop-expansion, computed using the parameters $b, w, s$, breaks down in the vicinity of a tricritical point, as it breaks down in the critical limit of very long chains. However, the renormalization group method [9], establishes the existence of a transformation, parametrized by a variable $m$ :

$$
(b, w, s) \rightarrow(b(m), w(m), m s),
$$

which leaves $\Gamma(f)$ invariant. $b(m), w(m)$ are certain functions of $m$, and obey the conditions $b(1)=b$, $w(1)=w\left({ }^{1}\right)$. The loop-expansion is valid if the coupling constants on the r.h.s. of (8) are taken at some particular large value of $m$, which depends on the concentration range. In the limit $m \rightarrow \infty$, one can determine the functions $b(m), w(m)[9,10]$ :

$$
w(m) \cong L(m), \quad b(m) \cong b w^{-p} L^{p}(m), \quad p=4 / 11,
$$

( $\left.{ }^{1}\right) a, f$ are also transformed under the action of the renormalization group into $a(m), f(m)$, not explicitly used here. with

$$
L(m)=\frac{16 \pi^{2}}{11} \frac{1}{|\ln m|} .
$$

One should note that the asymptotic behaviour of $w(m)$ is independent of $w$. This regime is reached if $[9,10]$

$$
1 \ll w \ln m .
$$

Let us now discuss the determination of the optimal value of $m$. There are two cases :

a) Dilute solutions. - The chains are characterized by the mean surface $S$. The scale $m s$ is fixed at $m s \cong S$. Thus :

$$
L(m) \cong L\left(S s^{-1}\right) .
$$

A more precise evaluation would add a constant term to the logarithm : this constant is not universal. According to (10), long chains in dilute solutions are tricritical if [4]

$$
1 \ll w \ln S s^{-1} .
$$


b) Semi-dilute solutions. - The condition is, say, $C_{\mathrm{p}} R^{3}>1$. Near $\theta: R^{2} \sim S$, and thus $C S^{1 / 2}>1$. For a given concentration $C$, we may define the correlation surface $\tilde{S},(\tilde{S}<S)$ along which the chains behave as in a dilute solution, by

$$
C \tilde{S}^{1 / 2} \cong 1 \text {. }
$$

The chains retain their tricritical behaviour on this scale $\tilde{S}$, and, as in (11), we set $m s \cong \widetilde{S}$, and

$$
L(m) \cong L\left(\tilde{S} s^{-1}\right) .
$$

$\tilde{S}$ is smaller than $S$ but must still be much larger than the minimal surface $s$. More precisely, the tricritical condition is (Eq. (10)) $1 \ll w \ln \tilde{S}^{-1}$. Using (13), (9b), we obtain

$$
\begin{gathered}
L(m) \cong L\left(C^{2} s\right), \\
1 \ll w\left|\ln C^{2} s\right| \text { with } C^{2} s \ll 1 .
\end{gathered}
$$

Thus, polymeric liquids $\left(C^{2} s \cong 1\right)$ are not expected to exhibit a tricritical behaviour.

3. Dilute solution near their flory point. - One can see from (2a) and (6) that $C \cong f^{2}$. Then using also (2b), the dilute solution condition $C^{2} \ll S^{-1}$ becomes $f^{4} \ll a$. In this limit $f \rightarrow 0, \Gamma(f)$ (Eq. (5)) may be expanded in powers of $f$ (or $C$ ). Eliminating the variables $a, f$ in the eq. (2) gives the one-loop expansion for $\Pi$ :

$$
\begin{aligned}
\Pi \beta=C_{\mathrm{p}}+\frac{1}{8} b(m) & C^{2}+\frac{1}{24} w(m) C^{3}- \\
& -\frac{1}{16 \pi} w(m)\left(\frac{S}{2}\right)^{-1 / 2} C^{2},
\end{aligned}
$$

where the $m$-dependent parameters (8) are used. The square radius (3) is computed by expanding (5), (7) in powers of $f$, and reads :

$$
\begin{array}{r}
R^{2}=d S\left\{1+\frac{7^{2}}{2^{6} 11 \pi^{2}} w(m)+\frac{1}{8 \pi}\left(\frac{S}{2}\right)^{1 / 2} \times\right. \\
\left.\times\left[b(m)+\frac{1}{2} w(m) C\right]\right\} .
\end{array}
$$

Actually, the first correction, proportional to $w(m)$ is obtained at the two-loop level only. Using eqs. $(9 a)$, (11) :

$$
\begin{aligned}
& \Pi \beta=C_{\mathrm{p}}+\left[\frac{1}{8} b w^{-p} L^{p}(S)-\frac{1}{16 \pi} L(S)\left(\frac{S}{2}\right)^{-1 / 2}\right] C^{2}+\frac{1}{24} L(S) C^{3} \\
& R^{2} \cong 3 S\left\{1+\frac{7^{2}}{2^{6} 11 \pi^{2}} L(S)+\frac{1}{8 \pi}\left(\frac{S}{2}\right)^{1 / 2}\left[b w^{-p} L^{p}(S)+\frac{1}{2} L(S) C\right]\right\}
\end{aligned}
$$

where $L(S)=\frac{16 \pi^{2}}{11}\left(\ln S s^{-1}\right)^{-1}$. The scaling laws (16a), (17a) are universal on the $\theta$-line $b=0$. M. J. Stephen [4] has found a similar first correction term in (17a) but with a different coefficient [13].

For finite chains of surface $S$, we define two effective $\theta$ temperatures : $T_{S}$, where the second virial term in (16a) vanishes, and $T_{S}^{\prime}$ where, in the limit $C \rightarrow 0$, $R^{2}=3 S$ exactly. Then, using (4), $(9 b)$ :

$$
\begin{aligned}
A \theta^{-1}\left|T_{S}-\theta\right| & \sim A \theta^{-1}\left|T_{S}^{\prime}-\theta\right| \\
& \sim w^{p} S^{-1 / 2}(\ln S)^{p-1}
\end{aligned}
$$

where $p-1=-7 / 11$, and $T_{S}^{\prime}<\theta<T_{S}$.

The size of the tricritical domain can be estimated with the help of figure 2. The scaled contribution

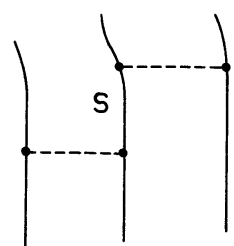

a)

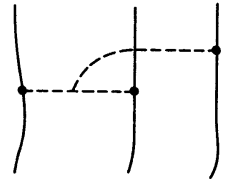

b)
Fig. 2. - Comparison of the three-chain interactions induced by two- and three-monomer interactions. for $a)$ is of order $S b^{2}(m)$, while that for $b$ ) is proportional to $w(m)$. Tricritical behaviour occurs when

$$
S b^{2}(m) \ll w(m) .
$$

Using eqs. (9), (4) :

$$
\begin{gathered}
A \theta^{-1}|T-\theta| \ll w^{p} S^{-1 / 2}(\ln S)^{p-1 / 2}, \\
p-1 / 2=-3 / 22 .
\end{gathered}
$$

This tricritical range of temperature (Fig. 3, region $\mathrm{D}$ ) agrees with a similar result by $\mathrm{P}$. G. de Gennes [7] (see also Ref. [6]). (18) is consistent with (19a).

Let us finally make precise the dilute solution condition. This condition is that in (16a), the perfect gas term dominates the three-chain term (the second virial term being neglected in the $\theta$-domain). Thus :

$$
C<(\ln S)^{1 / 2} S^{-1 / 2} .
$$

4. Semi-dilute solutions. - When $S$ is too large to satisfy (20), we are in the regime of semi-dilute solutions. According to (20), the correlation surface $\tilde{S}$ (Eq. (13)) is more precisely estimated by

$$
C\left|\ln C^{2} s\right|^{-1 / 2} \tilde{S}^{1 / 2} \cong 1 \text {. }
$$




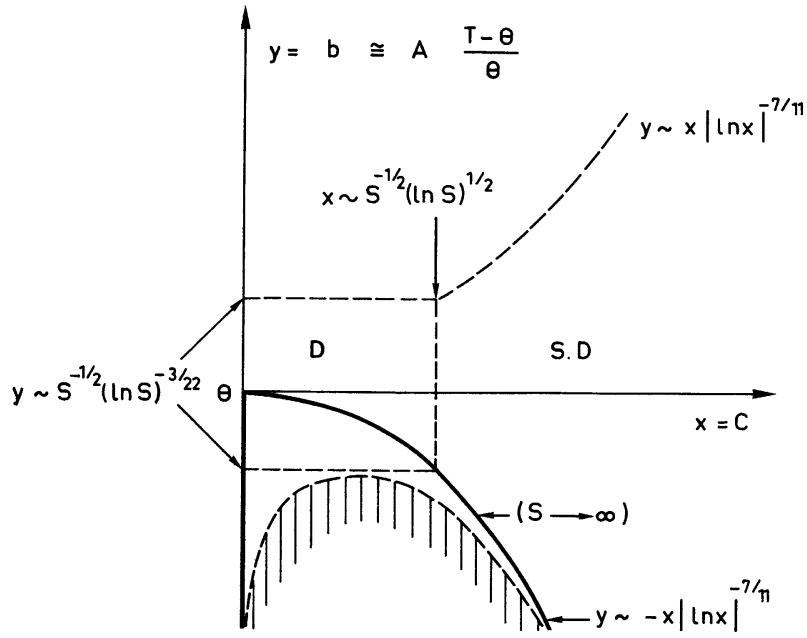

Fig. 3. - The tricritical Flory domain for a solution of chains of surface $S=N l^{2}$ (dotted lines). $D=$ dilute, S.D. $=$ semi-dilute. Coexistence curve for finite $S$ has been drawn on the figure but its precise form has not been calculated. We know only the shape of the coexistence curve when $S \rightarrow \infty$ (solid line).

The limit $S \rightarrow \infty, C$ constant, belongs to the semidilute range. Here it is no longer possible to expand $\Gamma(f)$ in powers of $f$. Instead, we note that (2), (6) give at first-order

$$
f^{-1} \frac{\partial}{\partial f} \Gamma_{0}(f)=(S / 2)^{-1}
$$

Therefore the semi-dilute range will be characterized by

$$
f^{-1} \frac{\partial \Gamma_{0}}{\partial f} \rightarrow 0
$$

In this limit, eqs. (2), (5), (6) give the one-lopp expansions

$\Pi \beta=C_{\mathrm{p}}+\frac{1}{8} b(m) C^{2}+\frac{1}{24} w(m) C^{3}+\delta \Pi \beta$,

where

(i) $\delta \Pi \beta \cong-\frac{1}{24 \pi} b^{3 / 2}(m) C^{3 / 2}$

or

$$
\text { (ii) } \delta \Pi \beta \cong-\frac{1}{2^{1 / 2} 12 \pi} w^{3 / 2}(m) C^{3}
$$

(i) is valid if $b(m)>w(m) C$ and (ii) in the opposite case. $(21$ (i)) is reminiscent of a result by S. F. Edwards [14], corrected by M. A. Moore [12]. The square radius (3) is obtained in the same way, by using (2), (5), (7) :

$$
R^{2}=d S\left\{1+\frac{1}{3 \pi}\left[\frac{1}{2} w(m)+b(m) C^{-1}\right]^{1 / 2}\right\} .
$$

It coincides with a result of Edwards [14], whereas that of Moore et al. [12] contains a different coefficient. The contribution from $(7 b)$ was not taken into account there.
(21) and (22) with (9a), (14) give the tricritical scaling laws :

$$
\begin{aligned}
& \Pi \beta \cong \frac{1}{24} L\left(C^{2}\right) C^{3}+\frac{1}{8} b w^{-p} L^{p}\left(C^{2}\right) C^{2} \\
& R^{2} \cong 3 S\left\{1+\frac{1}{3 \pi} \times\right. \\
& \times {\left.\left[\frac{1}{2} L\left(C^{2}\right)+b w^{-p} L^{p}\left(C^{2}\right) C^{-1}\right]^{1 / 2}\right\} }
\end{aligned}
$$

where

$$
L\left(C^{2}\right)=\frac{16 \pi^{2}}{11}\left|\ln C^{2} s\right|^{-1} .
$$

In (21a), we kept only the dominant terms of (21). On the $\theta$-line $b=0$, these scaling laws are universal. The size of the tricritical semi-dilute domain is obtained by the argument of eq. (19) figure 2 , used for the correlation surface $\tilde{S}: \widetilde{S} b^{2}(m) \ll w(m)$. (13a), (9) give, with (4) :

$$
A \theta^{-1}|T-\theta| \ll w^{p} C\left|\ln C^{2} s\right|^{p-1} .
$$

This defines the region S.D. of the $(C, T)$ diagram (Fig. 3).

5. The asymptotic coexistence curve. - At temperatures below $\theta$, the polymer system separates into two phases, a solution and a precipitate. In the $(C, T)$ diagram (Fig. 3) part of the coexistence curve (in the limit of infinite chains) is the half axis $C=0, T<\theta$, where $\Pi=0$. Because the two phases coexist at the same osmotic pressure, the precipitate also has $\Pi=0$. Setting this condition in (21a), we find, with (4), (9) :

$$
\theta^{-1}(T-\theta)=-A^{\prime} w^{p} C\left|\ln C^{2} s\right|^{p-1},
$$

where

$$
A^{\prime}=\frac{1}{3}\left(\frac{16 \pi^{2}}{11}\right)^{p-1} A^{-1}, \text { and } p-1=-\frac{7}{11} \text {. }
$$

The finite slope generally assumed for the coexistence curve at $S=\infty$, is not correct. The curve, shown in figure 3 , has zero slope at $C=0$, a feature typical of tricritical phenomena, which might be interesting to see.

6. Specific heat. - Numerical simulations [15] suggest an anomaly in the specific heat. It corresponds to the self energy of a chain, which can be calculated from the Lagrangian theory. We find a peak in the specific heat, with a peak value :

$$
C_{v} \cong c C\left(\ln S s^{-1}\right)^{1-2 p} \text { for a dilute solution, }
$$
and

$$
C_{v} \cong c C\left|\ln C^{2} s\right|^{1-2 p} \text { for a semi-dilute solution . }
$$

$c$ is a coefficient depending on $g, w, T$. The exponent $1-2 p=3 / 11$ agrees with that of de Gennes [7].

Acknowledgments. - The author is grateful to J. des Cloizeaux for many interesting discussions and remarks, and to M.E. Peskin for an helpful reading of the manuscript. 


\section{References}

[1] De Gennes, P. G., Phys. Lett. 38A (1972) 339.

[2] Des Cloizeaux, J., J. Physique 36 (1975) 281.

[3] De Gennes, P. G., J. Physique Lett. 36 (1975) L-55.

[4] Stephen, M. J., Phys. Lett. 53A (1975) 363.

[5] Daoud, M., Jannink, G., J. Physique 37 (1976) 973.

[6] Oono, Y., Oyama, T., J. Phys. Soc. Japan 44 (1978) 301.

[7] De Gennes, P. G., J. Physique Lett. 39 (1978) L-299.

[8] Duplantier, B., C.R. Hebd. Séan. Acad. Sci. Paris 290B (1980) 199.

[9] Wohrer, M., Thèse 3e cycle, Université Paris-VI (1976).

[10] Stephen, M. J., Abrahams, E., Straley, J. P., Phys. Rev. B 12 (1975) 256.

[11] The vertex function, transverse with respect to the $j=1$ direction, is defined by

$$
\delta\left(k+k^{\prime}\right) \Gamma_{\mathrm{T}}^{(2)}(k ; f)=\partial / \partial f^{i}\left(k^{\prime}\right) \partial /\left.\partial f^{i}(k) \Gamma\{\mathbf{f}\}\right|_{\mathbf{f}=f},
$$

with $i \neq 1$ and where $\Gamma\{\mathbf{f}\}$ has been generalized to depend on a spatially varying field $f^{i}(k)$.

[12] Moore, M. A., J. Physique 38 (1977) 265 ;

Moore, M. A., Al Noaimi, G. F., J. Physique 39 (1978) 1015.

[13] In reference [4], Stephen finds $R^{2}=3 S\left(1+\frac{37}{3(11)^{2}}(\ln S)^{-1}\right)$ whereas our result $(17 a)$, with $(9 a)$, gives a correction $\frac{49}{4(11)^{2}}(\ln S)^{-1}$. We believe that his second eq. (5) should involve $\left(\ln k^{2}\right)^{-2}$ instead of $\left(\ln k^{2}\right)^{-1}$, which just explains the slight discrepancy.

[14] Edwards, S. F., J. Phys. A 8 (1975) 1670.

[15] Domb, C., Polymer 15 (1974) 259;

Rapaport, D. C., Phys. Lett. 48A (1974) 339, J. Phys. A 10 (1977) 637. 